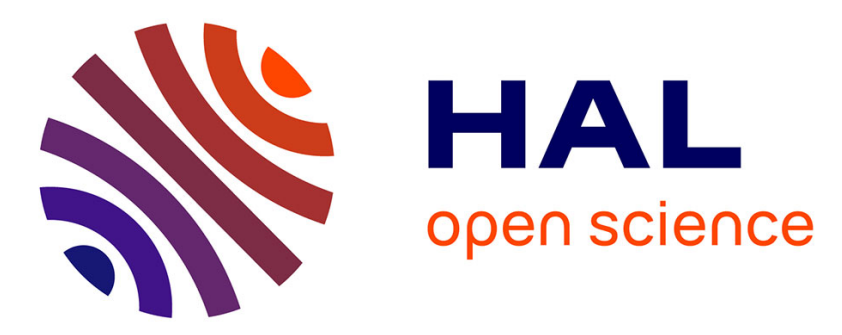

\title{
Efficacité du cerclage préventif indiqué sur des antécédents obstétricaux en cas de grossesse gémellaire
}

\author{
M. Métairie, D. Korb, C. Morin, T. Schmitz, O. Sibony
}

\section{To cite this version:}

M. Métairie, D. Korb, C. Morin, T. Schmitz, O. Sibony. Efficacité du cerclage préventif indiqué sur des antécédents obstétricaux en cas de grossesse gémellaire. Gynécologie Obstétrique Fertilité \& Sénologie, 2019, 47, pp.286 - 290. 10.1016/j.gofs.2018.12.009 . hal-03486623

\section{HAL Id: hal-03486623 \\ https://hal.science/hal-03486623}

Submitted on 21 Dec 2021

HAL is a multi-disciplinary open access archive for the deposit and dissemination of scientific research documents, whether they are published or not. The documents may come from teaching and research institutions in France or abroad, or from public or private research centers.
L'archive ouverte pluridisciplinaire HAL, est destinée au dépôt et à la diffusion de documents scientifiques de niveau recherche, publiés ou non, émanant des établissements d'enseignement et de recherche français ou étrangers, des laboratoires publics ou privés.

\section{(ㄷ)(1) $\$$}

Distributed under a Creative Commons Attribution - NonCommerciall 4.0 International 
Titre : Efficacité du cerclage préventif indiqué sur des antécédents obstétricaux en cas de grossesse gémellaire

Titre en anglais : Effectiveness of preventive cervical cerclage to prevent preterm birth in women with twin gestation with obstetrical history of late pregnancy loss or preterm birth.

Auteurs : Marie Métairie ${ }^{1}$, Diane Korb ${ }^{1,2}$, Cécile Morin ${ }^{1}$, Thomas Schmitz $^{1,2}$, Olivier Sibony ${ }^{1}$

\section{Affiliations :}

1. Hopital Robert Debré, APHP, Paris, France

2. INSERM U1153, Equipe EPOPé, CRESS, DHU Risques et grossesse, Université Paris Descartes, Paris, France

\section{Auteur correspondant :}

Marie Métairie

Maternité Robert Debré, 48 boulevard Sérurier 75019 Paris

marie metairie@hotmail.fr

0633038918

Travail présenté au congrès de la SFMP à Lyon le 19/10/2017

Travail présenté au congrès du CNGOF à Lille le 06/12/2017 


\section{INTRODUCTION}

2 Le taux de grossesses gémellaires a augmenté ces dernières années. Il est estimé à 1,8\% en

3 France. Le taux de prématurité en cas de grossesse gémellaire est de 47,5\%, alors qu'il est de

$4 \quad 6,0 \%$ pour les singletons [1]. Par ailleurs, les antécédents de fausse couche tardive ou

5 d'accouchement prématuré sont des facteurs de risque de prématurité.

6 Ainsi, les grossesses gémellaires avec antécédent de fausse couche tardive et/ou

7 d'accouchement prématuré sont à haut risque d'accouchement prématuré, du fait de la 8 présence de deux facteurs de risque majeurs.

9 En cas de grossesse gémellaire sans antécédents obstétricaux, plusieurs moyens de prévention 10 de la prématurité ont été étudié, notamment le pessaire [3-5], la progestérone [6,7] ou la 11 surveillance cervicale échoguidée avec cerclage écho-indiqué [8-10]. Ces derniers ont pour la 12 plupart été étudiés dans une population de grossesses gémellaires asymptomatiques avec 13 découverte échographique de col court. Aucune étude n’a mis en évidence une efficacité de ces méthodes pour prévenir la prématurité en cas de grossesse gémellaire.

Très peu d'étude se sont intéressées aux moyens préventifs en cas de grossesse gémellaire et d'antécédents obstétricaux. En cas de grossesse monofoetale avec antécédents obstétricaux, le cerclage semble être efficace dans la prévention de la prématurité en cas antécédents répétés. Se pose la question d'appliquer les indications de cerclage des singletons aux grossesses gémellaires. Une seule analyse ancienne datant de 1993 issue d'un essai randomisé a étudié en sous-groupe le cerclage préventif des grossesses gémellaires avec antécédent de fausse couche tardive et/ou d'accouchement prématuré [11]. Elle n'a pas montré de différence significative 22 sur le taux d'accouchement avant 34SA. Cependant, cette étude avait des limites, notamment un très faible nombre de cas. 
L'objectif de cette étude était d'évaluer l'efficacité du cerclage préventif indiqué sur des antécédents obstétricaux de fausse couche tardive ou d'accouchement prématuré en cas de grossesse gémellaire.

\section{METHODES}

Il s'agissait d'une étude rétrospective de cohorte hospitalière de type exposées/non exposées.

Toutes les patientes présentant une grossesse gémellaire ayant accouché, dans notre maternité universitaire de type 3, entre 2002 et 2017 et ayant au moins un antécédent de fausse couche tardive et/ou d'accouchement prématuré spontané avant 34 SA ont été incluses $(n=113)$ (figure 1). Ont été exclues les patientes ayant des antécédents de prématurité induite $(\mathrm{n}=44)$. Deux groupes d'étude ont été définis : les patientes ayant été cerclées préventivement $(\mathrm{n}=20)$ et les patientes n'ayant pas été cerclées préventivement $(n=49)$. Les patientes qui n'étaient pas cerclées n'ont pas eu de surveillance particulière, notamment pas de surveillance cervicale échographique systématique.

La décision du cerclage relevait d'une discussion collégiale en staff, en tenant compte des antécédents des patientes et de leur histoire obstétricale. Le cerclage était réalisé selon la technique de Mac Donald. Dans notre centre, la pratique du cerclage répond à des indications larges avec réalisation d'un cerclage préventif dès un antécédent de fausse couche tardive ou d'accouchement prématuré spontané si l'histoire clinique est évocatrice d'une béance cervicale. Le cerclage est réalisé après l'échographie du premier trimestre. Sa pratique est associée à du repos au lit non strict et à un arrêt de travail mais aucun traitement complémentaire n'est prescrit par ailleurs (comme la progestérone). La suite de la prise en charge consiste en une surveillance clinique mensuelle associée à un prélèvement vaginal et traitement antibiotique si présence de Gardnerella Vaginalis. 
Les données de cette étude sont issues de la base de données informatisée du service, remplie prospectivement à chaque consultation, à chaque passage aux urgences, à chaque hospitalisation et en salle de travail. L'informatisation de ces données était faite via le logiciel de la maternité par le professionnel de santé responsable de la patiente, sage-femme ou médecin. L'exactitude des données a été vérifiée lors du staff le lendemain de l'accouchement.

Les caractéristiques maternelles étudiées étaient l'âge maternel, la parité, le tabagisme, l'origine géographique, la catégorie socio-professionnelle, l'indice de masse corporelle, et les antécédents d'hypertension artérielle et de diabète. Les caractéristiques obstétricales étudiées étaient le type de procréation et les antécédents de pathologie hypertensive gravidique, d'accouchement prématuré, de cerclage, de fausse couche tardive ainsi que le nombre d'accouchement voie basse et de césarienne antérieur.

La distinction a été faite entre les antécédents de prématurité induite et spontanée après relecture de chaque dossier. Les antécédents d'accouchement prématuré ont été classés en fonction de leur nombre, de 0 à $\geq 3$. Par ailleurs, les caractéristiques de l'accouchement, notamment le mode d'entrée en travail, le taux de prématurité induite versus spontanée, la voie d'accouchement et le taux d'hémorragie du post-partum, ont été analysées.

Le critère de jugement principal était le taux d'accouchement prématuré avant 34 SA. Les critères de jugement secondaires étaient le taux d'accouchement prématuré avant $32 \mathrm{SA}$, avant 28 SA et avant 24 SA, ainsi que les issues néonatales.

Des analyses univariées ont été réalisées pour l'ensemble des caractéristiques maternelles, obstétricales et néonatales avec un test du Chi2 ou de Fischer. Des analyses multivariées à l'aide de modèles de régression logistiques ont été réalisées en prenant en compte comme 
71 facteur de confusion le nombre d'antécédents de fausse couche tardive et d'accouchement

72 prématuré. L’ensemble des analyses a été effectué au moyen du logiciel STATA 13.

\section{RESULTATS}

75 Sur les 1972 grossesses gémellaires ayant accouché dans le service entre 2002 et 2017, 113 $76(5.7 \%)$ présentaient au moins un antécédent de fausse couche tardive ou d'accouchement 77 prématuré avant 34 SA. Après exclusion de 44 (38.9\%) patientes présentant uniquement des antécédents de prématurité induite, $69(61.1 \%)$ patientes ont été incluses. Le groupe «cerclage préventif» était constitué de 20 femmes (29.0\%) et le groupe «absence de cerclage préventif » de 49 femmes $(71.0 \%)$ (Figure 1).

Les deux groupes étaient comparables concernant les caractéristiques maternelles (Tableau 1), hormis l'origine géographique.

Concernant les antécédents obstétricaux, les femmes du groupe «cerclage préventif» 84 présentaient significativement plus d'antécédents de fausses couches tardives $(80.0 \%$ versus $8553.1 \%, \mathrm{p}=0.038)$. On notait de façon significative plus d'antécédents de cerclage dans le groupe « cerclage préventif » $(20.0 \%$ versus $2.0 \%, \mathrm{p}=0.03)$ (Tableau 1$).$

87 Le terme médian du cerclage préventif était de 15 SA et 4 jours. Le terme médian du décerclage était de $35 \mathrm{SA}$ et 3 jours. Quatre (8.2\%) patientes du groupe « absence de cerclage préventif » ont été cerclées à chaud du fait de modifications cervicales.

Concernant les caractéristiques de la grossesse, il n'y avait pas de différence significative entre les deux groupes sur le plan de la chorionicité avec une majorité de grossesse bichoriale biamniotique. Le taux d'hospitalisation pour menace d'accouchement prématuré ne différait 
94 rupture prématurée des membranes $(25.0 \%$ vs $8.2 \%$, p=0.06). Il n'existait pas de différence 95 significative sur le recours à la tocolyse, ou à la corticothérapie maturative (Tableau 2).

Concernant le critère de jugement principal, il n'existait pas de différence significative entre les deux groupes $(45.0 \%$ versus $44.9 \%, \mathrm{p}=0.99$, OR brut : 1.00 [0.35-2.83]). Ces résultats étaient inchangés après ajustement sur le nombre d'antécédents de fausse couche tardive et d'accouchement prématuré (OR ajusté : 1.06 [0.33-3.44]).

Il n'existait pas de différence significative concernant le caractère induit ou spontané de la prématurité avant 34 SA dans les deux groupes (100\% de prématurité spontanée dans le groupe « cerclage préventif » versus $81.8 \%$ dans le groupe « absence de cerclage $» \mathrm{p}=0.17$ ).

La répartition des termes d'accouchement ne différait pas entre les deux groupes (Tableau 3).

Il n'existait pas non plus de différence significative quant au déroulement du travail ou quant aux complications du post-partum (Tableau 4).

Il n'existait pas de différence significative entre les deux groupes concernant les issues néonatales, notamment pour le $\mathrm{pH}$, l'APGAR $<4$ à 5 minutes, la réanimation néonatale (Tableau 5).

\section{DISCUSSION}

Dans cette étude de cohorte rétrospective de grossesses gémellaires il n'est pas mis en évidence de différence significative sur le taux de prématurité avant 34 SA en cas de cerclage préventif indiqué sur des antécédents obstétricaux comparativement à l'absence de cerclage.

Ce résultat doit être nuancé par le fait que les femmes du groupe cerclage avaient des antécédents obstétricaux plus sévères que les femmes n'ayant pas eu de cerclage. 

cas de grossesses gémellaires date de 1993. Il s'agissait d'une étude en sous-groupe de 28 grossesses gémellaires, dont 12 avaient été cerclées comparativement à 16 non cerclées, étudiant l'efficacité du cerclage dans la prévention de la prématurité en cas d'antécédents gynéco-obstétricaux. Il n'existait pas de différence significative sur le taux d'accouchement avant $34 \mathrm{SA}, \mathrm{RR}=0,27$, IC 95\% [0,04-1,99]. Sa méthodologie n'a pas permis de distinguer les antécédents obstétricaux des antécédents gynécologiques [11].

124 Ces résultats avaient été repris dans une méta-analyse de 2014 soulignant le manque de puissance compte tenu du faible nombre de patientes présentant des grossesses gémellaires [12].

127 En 2018, Han et al ont publié une étude de cohorte rétrospective analysant l'efficacité du 128 cerclage des grossesses gémellaires, comparativement à l'absence de cerclage, en cas 129 d'antécédents obstétricaux, de col échographiquement court ou de dilatation cervicale $\geq 1 \mathrm{~cm}$. 130 Parmi les 96 patientes cerclées, seulement 6 présentaient uniquement des antécédents obstétricaux. L'efficacité du cerclage indiqué uniquement sur antécédents obstétricaux n'a donc pas pu être étudiée [13].

133 Ainsi, il n'existe, à notre connaissance, aucune publication traitant du cerclage préventif sur 134 antécédent obstétrical seul en cas de grossesse gémellaire, ne rendant pas possible la comparaison de nos résultats avec la littérature. En cas de grossesse gémellaire, dans les autres indications possibles, l'efficacité du cerclage est discutée. En effet, il n'a pas été montré de bénéfice en l'absence d'antécédent $(14,15)$, ou en cas de cerclage échoguidé (12, 16). 
Concernant l'efficacité du cerclage à chaud des grossesses gémellaires, les données disponibles montraient une diminution significative du taux d'accouchement prématuré, quel que soit le terme, sans réduction de la mortalité périnatale. Néanmoins, seules les femmes cerclées avaient reçu des traitements concomitants par Indométacine ou par antibiotique, associés à une prolongation de la grossesse, responsable d'un biais majeur et à prendre en compte dans l'interprétation des résultats [17].

Notre étude présente plusieurs forces. Le nombre relativement important de patientes incluses pour la problématique traitée, le caractère unicentrique ayant permis une homogénéisation des pratiques et des prises en charge des femmes. On peut également souligner que toutes les données concernant les patientes ont été recueillies de manière prospective, permettant d'assurer une bonne validité des données.

De même, la distinction entre la prématurité induite et spontanée, que cela soit au niveau des antécédents obstétricaux ou au cours de la grossesse étudiée, a pu être faite, ce qui n'ai quasiment jamais précisé lors des études des moyens de prévention de la prématurité en cas d'antécédents obstétricaux. partiellement pris en compte par l'ajustement. L'indication du cerclage a sans doute été portée pour des antécédents plus graves : plus d'antécédents de cerclage à chaud lors des précédentes grossesses, plus d'antécédents de fausses couches tardives. Par ailleurs, certaines patientes n'ont pas été cerclées alors même qu'elles auraient été candidates à un cerclage du fait d'une prise en charge tardive dans notre centre. Ainsi l'absence de bénéfice du cerclage peut être expliquée par un niveau de risque initial plus élevé dans le groupe cerclage. 
164 Une autre limite est que, malgré le nombre important de patientes incluses pour la 165 problématique traitée, cette étude manque de puissance du fait des faibles effectifs de chaque 166 groupe. Le design rétrospectif et le fait que les populations ne soient pas comparables 167 limitent l'interprétation des résultats.

Bien que cela soit probablement très difficile à mettre en œuvre en pratique, un essai contrôlé randomisé prospectif serait intéressant à réaliser au sein d'une population plus large,

171 afin de statuer sur l'intérêt du cerclage dans la prévention de la prématurité en cas de 172 grossesse gémellaire avec antécédent obstétrical.

\section{CONCLUSION}

Cette étude met en évidence, en cas de grossesse gémellaire avec antécédents 176 obstétricaux de fausse couche tardive ou de prématurité, un taux d'accouchement prématuré 177 similaire entre le groupe des femmes cerclées ayant plus d'antécédents de FCT et les femmes 178 du groupe non cerclées. En attendant d'autres études de méthodologie plus robuste sur le 179 sujet, l'indication du cerclage dans ces situations doit être étudiée au cas par cas. 
Les auteurs déclarent ne pas avoir de liens d'intérêts.

\section{REFERENCES}

1. Enquêtes Nationales Périnatales 2016.

2. Easter S, Little S, Robinson J, Mendez-Figueroa H, Chauhan S. Obstetric History and Likelihood of Preterm Birth of Twins. Am J Perinatol [Internet]. 2018 Jan 5 [cited 2018 Mar 23]; Available from: http://www.thieme-connect.de/DOI/DOI?10.1055/s-0037-1617758

3. Goya M, de la Calle M, Pratcorona L, Merced C, Rodó C, Muñoz B, et al. Cervical pessary to prevent preterm birth in women with twin gestation and sonographic short cervix: a multicenter randomized controlled trial (PECEP-Twins). Am J Obstet Gynecol. 2016 Feb;214(2):145-52.

4. Nicolaides KH, Syngelaki A, Poon LC, de Paco Matallana C, Plasencia W, Molina FS, et al. Cervical pessary placement for prevention of preterm birth in unselected twin pregnancies: a randomized controlled trial. Am J Obstet Gynecol. 2016 Jan;214(1):3.e1-3.e9. 5. Berghella V, Dugoff L, Ludmir J. Prevention of preterm birth with pessary in twins (PoPPT): a randomized controlled trial: Prevention of preterm birth with pessary in twins. Ultrasound Obstet Gynecol. 2017 May;49(5):567-72.

6. Schuit E, Stock S, Rode L, Rouse D, Lim A, Norman J, et al. Effectiveness of progestogens to improve perinatal outcome in twin pregnancies: an individual participant data meta-analysis. BJOG Int J Obstet Gynaecol. 2015 Jan;122(1):27-37.

7. Romero R, Conde-Agudelo A, El-Refaie W, Rode L, Brizot ML, Cetingoz E, et al. Vaginal progesterone decreases preterm birth and neonatal morbidity and mortality in women with a twin gestation and a short cervix: an updated meta-analysis of individual patient data: 
Vaginal progesterone decreases preterm birth in twin gestation with short cervix. Ultrasound

206 Obstet Gynecol. 2017 Mar;49(3):303-14.

207 8. Gordon MC, McKenna DS, Stewart TL, Howard BC, Foster KF, Higby K, et al. 208 Transvaginal cervical length scans to prevent prematurity in twins: a randomized controlled 209 trial. Am J Obstet Gynecol. 2016 Feb;214(2):277.e1-277.e7.

210 9. Saccone G, Rust O, Althuisius S, Roman A, Berghella V. Cerclage for short cervix in 211 twin pregnancies: systematic review and meta-analysis of randomized trials using individual 212 patient-level data. Acta Obstet Gynecol Scand. 2015 Apr;94(4):352-8.

213 10. Houlihan C, Poon LCY, Ciarlo M, Kim E, Guzman ER, Nicolaides KH. Cervical 214 cerclage for preterm birth prevention in twin gestation with short cervix: a retrospective 215 cohort study. Ultrasound Obstet Gynecol Off J Int Soc Ultrasound Obstet Gynecol. 2016 216 Dec;48(6):752-6.

217 11. MRC/RCOG Working Party on Cervical Cerclage, Macnaughton MC, Chalmers IG, 218 Dubowitz V, Dunn PM, Grant AM, et al. Final report of the Medical Research Council/Royal 219 College of Obstetricians and Gynaecologists Multicentre Randomised Trial of Cervical 220 Cerclage. BJOG Int J Obstet Gynaecol. 1993 Jun;100(6):516-23.

221 12. Rafael TJ, Berghella V, Alfirevic Z. Cervical stitch (cerclage) for preventing preterm 222 birth in multiple pregnancy. Cochrane Pregnancy and Childbirth Group, editor. Cochrane 223 Database Syst Rev [Internet]. 2014 Sep 10 [cited 2018 Mar 22]; Available from: 224 http://doi.wiley.com/10.1002/14651858.CD009166.pub2

225 13. Han MN, O’Donnell BE, Maykin MM, Gonzalez JM, Tabsh K, Gaw SL. The impact 226 of cerclage in twin pregnancies on preterm birth rate before 32 weeks. J Matern Fetal 227 Neonatal Med. 2018 Jan 23;1-9. 
228 14. Seki H, Kuromaki K, Takeda S, Kinoshita K. Prophylactic cervical cerclage for the 229 prevention of early premature delivery in nulliparous women with twin pregnancies. J Obstet 230 Gynaecol Res. 2000 Apr;26(2):151-2.

231 15. Eskandar M, Shafiq H, Almushait MA, Sobande A, Bahar AM. Cervical cerclage for 232 prevention of preterm birth in women with twin pregnancy. Int J Gynaecol Obstet Off Organ 233 Int Fed Gynaecol Obstet. 2007 Nov;99(2):110-2.

234 16. Berghella V, Odibo AO, To MS, Rust OA, Althuisius SM. Cerclage for short cervix 235 on ultrasonography: meta-analysis of trials using individual patient-level data. Obstet 236 Gynecol. 2005 Jul;106(1):181-9.

237 17. Roman A, Rochelson B, Martinelli P, Saccone G, Harris K, Zork N, et al. Cerclage in 238 twin pregnancy with dilated cervix between 16 to 24 weeks of gestation: retrospective cohort 239 study. Am J Obstet Gynecol. 2016 Jul;215(1):98.e1-98.e11. 


\section{Figure 1 : Population d'étude}

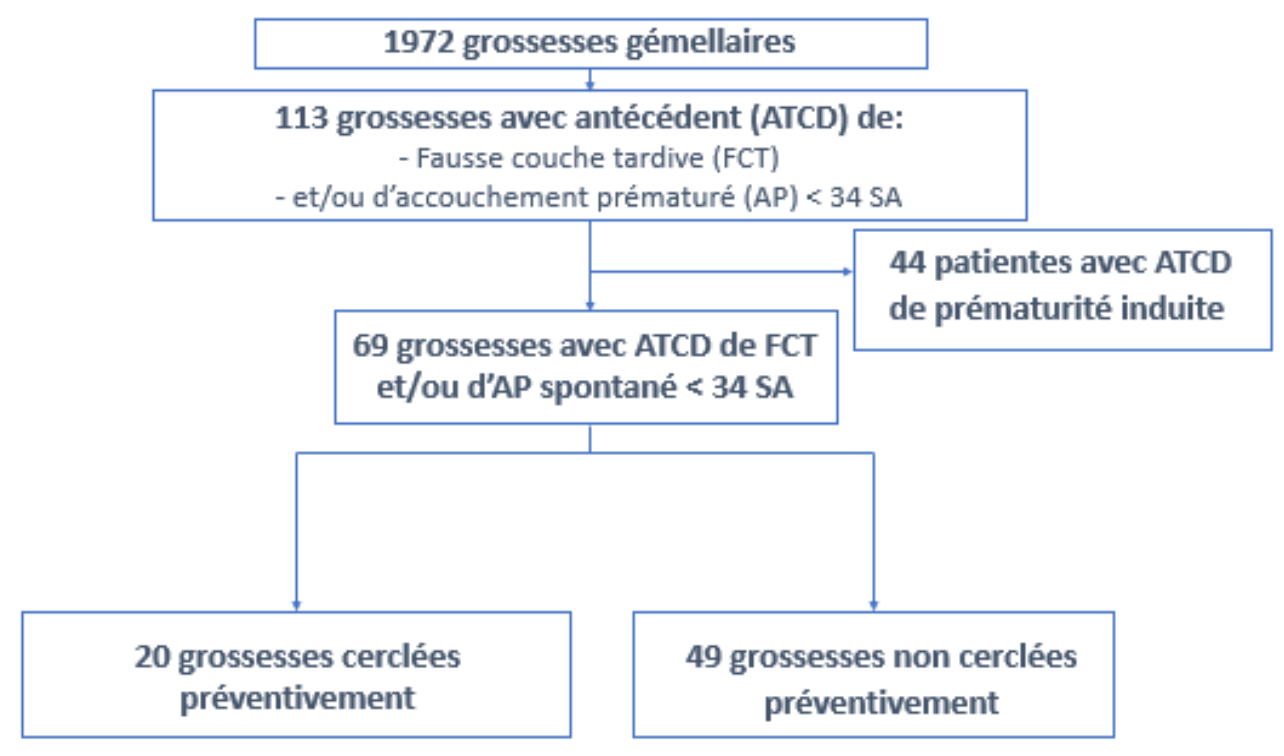




\section{Tableau 1 : Caractéristiques maternelles}

\begin{tabular}{|c|c|c|c|}
\hline & $\begin{array}{c}\text { Cerclage } \\
\mathrm{N}=20\end{array}$ & $\begin{array}{l}\text { Pas de cerclage } \\
\quad \mathrm{N}=49\end{array}$ & $\mathrm{p}$ \\
\hline & $\mathrm{N}(\%)$ & $\mathrm{N}(\%)$ & \\
\hline Age maternel (années) (moyenne \pm DS) & $30.0 \pm 5.8$ & $32.2 \pm 5.4$ & 0.14 \\
\hline$<30$ ans & $11(55.0)$ & $14(28.6)$ & 0.12 \\
\hline [30-39] ans & $8(40.0)$ & $31(63.3)$ & \\
\hline$\geq 40$ ans & $1(5.0)$ & $4(8.2)$ & \\
\hline Parité & & & 0.71 \\
\hline 1 & $1(5.0)$ & $1(2.0)$ & \\
\hline 2 & $5(25.0)$ & $10(20.4)$ & \\
\hline$\geq 3$ & $14(70.0)$ & $38(77.6)$ & \\
\hline Tabac avant la grossesse & $1(7.1)$ & $4(16.0)$ & 0.43 \\
\hline Origine géographique & & & 0.007 \\
\hline Caucasienne & $0(0.0)$ & $11(35.5)$ & \\
\hline Dom et Tom & $0(0.0)$ & $6(12.5)$ & \\
\hline Afrique & $14(93.3)$ & $14(45.2)$ & \\
\hline Afrique du Nord & $1(6.7)$ & $0(0.0)$ & \\
\hline Asie & $0(0.0)$ & $1(3.2)$ & \\
\hline Profession & & & 0.65 \\
\hline Cadre & $3(18.7)$ & $9(25.7)$ & \\
\hline Profession intermédiaire & $0(0.0)$ & $2(5.7)$ & \\
\hline Ouvrier & $7(43.8)$ & $15(42.9)$ & \\
\hline Sans emploi & $6(37.5)$ & $9(25.7)$ & \\
\hline $\begin{array}{l}\text { Index de masse corporelle }\left(\mathrm{kg} / \mathrm{m}^{2}\right) \\
(\text { moyenne } \pm \text { DS })\end{array}$ & $23.6 \pm 4.3$ & $24.9 \pm 5.3$ & 0.18 \\
\hline$<20$ & $2(10.0)$ & $5(12.8)$ & 0.32 \\
\hline [20-24] & $9(45.0)$ & $17(43.6)$ & \\
\hline [25-29] & $8(40.0)$ & $9(23.1)$ & \\
\hline$\geq 30$ & $1(5.0)$ & $8(20.5)$ & \\
\hline \multicolumn{4}{|l|}{ Antécédents maternels } \\
\hline Hypertension artérielle & $1(5.0)$ & $2(4.1)$ & 0.86 \\
\hline Diabète & $0(0.0)$ & $0(0.0)$ & \\
\hline Type de procréation & & & 0.004 \\
\hline Spontanée & $12(60.0)$ & $44(89.8)$ & \\
\hline Procréation médicalement assistée & $8(40.0)$ & $5(10.2)$ & 0.22 \\
\hline Fécondation in vitro & 5 & 1 & \\
\hline Stimulation ovarienne & 2 & 2 & \\
\hline Insémination artificielle & 1 & 1 & \\
\hline Don d'ovocyte & 0 & 1 & \\
\hline
\end{tabular}




\begin{tabular}{|c|c|c|c|}
\hline & $\begin{array}{l}\text { Cerclage } \\
\mathrm{N}=20\end{array}$ & $\begin{array}{l}\text { Pas de cerclage } \\
\quad \mathrm{N}=49\end{array}$ & $\mathrm{p}$ \\
\hline & $\mathrm{N}(\%)$ & $\mathrm{N}(\%)$ & \\
\hline Pathologie hypertensive gravidique & $0(0.0)$ & $2(4.1)$ & 0.36 \\
\hline Antécédent de césarienne & $1(5.0)$ & $5(10.2)$ & 0.49 \\
\hline Antécédent de cerclage & $4(20.0)$ & $1(2.0)$ & 0.03 \\
\hline Antécédent de prématurité spontanée & $11(55.0)$ & $30(61.2)$ & 0.63 \\
\hline Nombre d'AP ${ }^{\mathrm{a}}$ spontané (moyenne $\pm \mathrm{DS}$ ) & $0.7 \pm 0.8$ & $0.7 \pm 0.6$ & \\
\hline 0 & $11(55.0)$ & $22(44.9)$ & 0.39 \\
\hline 1 & $7(35.0)$ & $25(51.0)$ & \\
\hline 2 & $2(10.0)$ & $2(4.1)$ & \\
\hline$\geq 3$ & $0(0.0)$ & $0(0.0)$ & \\
\hline Antécédent de fausse couche tardive & $16(80.0)$ & $26(53.1)$ & 0.04 \\
\hline Nombre (moyenne \pm DS) & $1 \pm 0.7$ & $0.6 \pm 0.6$ & 0.09 \\
\hline 0 & $4(20.0)$ & $23(46.9)$ & \\
\hline 1 & $13(65.0)$ & $24(49.0)$ & \\
\hline 2 & $2(10.0)$ & $2(4.1)$ & \\
\hline$\geq 3$ & $1(5.0)$ & $0(0.0)$ & \\
\hline Antécédent de $\mathrm{FCT}^{\mathrm{b}}$ ou d'AP & $20(100.0)$ & $49(100.0)$ & \\
\hline 1 & $11(55.0)$ & $39(79.6)$ & 0.045 \\
\hline 2 & $6(30.0)$ & $9(18.4)$ & \\
\hline 3 & $3(15.0)$ & $1(2.0)$ & \\
\hline
\end{tabular}

${ }^{a}$ AP : Accouchement prématuré, ${ }^{b}$ FCT : Fausse couche tardive 


\section{Tableau 2 : Caractéristiques de la grossesse}

\begin{tabular}{|c|c|c|c|}
\hline & $\begin{array}{c}\text { Cerclage } \\
\mathrm{N}=20\end{array}$ & $\begin{array}{c}\text { Pas de cerclage } \\
\quad \mathrm{N}=49\end{array}$ & $\mathrm{p}$ \\
\hline & $\mathrm{N}(\%)$ & $\mathrm{N}(\%)$ & \\
\hline Chorionicité & & & 0.23 \\
\hline Monochoriale-monoamniotique & $0(0.0)$ & $0(0.0)$ & \\
\hline Monochoriale-biamniotique & $5(25.0)$ & $6(13.0)$ & \\
\hline Bichoriale-biamniotique & $15(75.0)$ & $40(87.0)$ & \\
\hline Prélèvement fotal invasif & $1(5.0)$ & $13(26.5)$ & 0.04 \\
\hline \multicolumn{4}{|l|}{ Complications de la grossesse } \\
\hline Hospitalisation pour MAP ${ }^{a}$ & $9(45.0)$ & $24(49.0)$ & 0.76 \\
\hline Hospitalisation pour RPM ${ }^{\mathrm{b}}$ & $5(25.0)$ & $4(8.2)$ & 0.06 \\
\hline Chorioamniotite (clinique, biologique) & $4(20.0)$ & $8(16.3)$ & 0.72 \\
\hline Diabète gestationnel & $3(15.0)$ & $2(4.1)$ & 0.11 \\
\hline Pathologie hypertensive gravidique & $0(0.0)$ & $1(2.0)$ & 0.52 \\
\hline $\mathrm{RCIU}^{\mathrm{c}}$ sur J1 et/ou J2 ${ }^{\mathrm{d}}$ & $2(10.0)$ & $5(10.2)$ & 0.98 \\
\hline Hospitalisation $<37 \mathrm{SA}$ & $17(85.0)$ & $36(73.5)$ & 0.30 \\
\hline Tocolyse IV ou PO & $15(75.0)$ & $25(51.0)$ & 0.07 \\
\hline Echappement à la tocolyse & $3(15.0)$ & $12(24.5)$ & 0.39 \\
\hline Corticothérapie maturative & $8(40.0)$ & $13(26.5)$ & 0.27 \\
\hline Transfert in utéro & $2(10.0)$ & $8(16.3)$ & 0.49 \\
\hline
\end{tabular}

${ }^{a}$ MAP : menace d'accouchement prématuré, ${ }^{b} R P M:$ rupture prématurée des membranes, ${ }^{c} R C I U$ : retard de croissance intra-utérin, ${ }^{d}$ J1 et/ou J2 : premier jumeau et/ou deuxième jumeau 


\section{Tableau 3 : Terme de l'accouchement}

\begin{tabular}{|c|c|c|c|}
\hline & $\begin{array}{c}\text { Cerclage } \\
\mathrm{N}=20\end{array}$ & $\begin{array}{c}\text { Pas de cerclage } \\
\quad \mathrm{N}=49\end{array}$ & $\mathrm{p}$ \\
\hline & $\mathrm{N}(\%)$ & $\mathrm{N}(\%)$ & \\
\hline Terme de naissance en $\mathrm{SA}^{\mathrm{a}}$ & & & 0.53 \\
\hline$\geq 37 \mathrm{SA}$ & $6(30.0)$ & $11(22.5)$ & \\
\hline [34-36] & $5(25.0)$ & $16(32.6)$ & \\
\hline [32-33] & $2(10.0)$ & $7(14.2)$ & \\
\hline [28-31] & $1(5.0)$ & $8(16.3)$ & \\
\hline [24-27] & $3(15.0)$ & $3(6.2)$ & \\
\hline$<24 \mathrm{SA}$ & $3(15.0)$ & $4(8.2)$ & \\
\hline Taux de prématurité < $34 \mathrm{SA}$ & $9(45.0)$ & $22(44.9)$ & 0.99 \\
\hline Induite & $0(0)$ & $4(18.2)$ & 0.17 \\
\hline Spontanée & $9(100)$ & $18(81.8)$ & 0.17 \\
\hline
\end{tabular}

${ }^{a}$ SA : Semaine d'aménorrhée 


\section{Tableau 4 : Caractéristiques de l'accouchement}

\begin{tabular}{|c|c|c|c|}
\hline & $\begin{array}{c}\text { Cerclage } \\
\mathrm{N}=20\end{array}$ & $\begin{array}{c}\text { Pas de cerclage } \\
\quad N=49\end{array}$ & $\mathrm{p}$ \\
\hline & $\mathrm{N}(\%)$ & $\mathrm{N}(\%)$ & \\
\hline \multicolumn{4}{|l|}{ Déroulement du travail } \\
\hline Ocytocine pendant le travail & $8(40.0)$ & $17(34.7)$ & 0.68 \\
\hline Rupture des membranes & & & 0.20 \\
\hline Spontanée & $12(60.0)$ & $37(75.5)$ & \\
\hline Artificielle & $8(40.0)$ & $12(24.5)$ & \\
\hline Couleur du LA ${ }^{1}$ pendant le travail & & & 0.54 \\
\hline Claire & $17(100.0)$ & $40(93.0)$ & \\
\hline Teintée & $0(0.0)$ & $1(2.3)$ & \\
\hline Méconiale & $0(0.0)$ & $2(4.7)$ & \\
\hline Chorioamniotite pendant travail & $4(20.0)$ & $8(16.3)$ & 0.71 \\
\hline Durée du travail (moyenne \pm DS) & $396 \pm 269$ & $248 \pm 211$ & 0.03 \\
\hline Voie d'accouchement & & & 0.74 \\
\hline Voie basse & $16(80.0)$ & $36(73.5)$ & 0.79 \\
\hline Voie basse spontanée & $16(100.0)$ & $34(94.4)$ & \\
\hline Voie basse instrumentale & $0(0.0)$ & $2(5.6)$ & \\
\hline Césarienne & $4(20.0)$ & $13(26.5)$ & 0.57 \\
\hline Indication de césarienne & & & 0.49 \\
\hline $\mathrm{ARCF}^{2}$ & $1 / 4$ & $7 / 13$ & \\
\hline Stagnation & $1 / 4$ & $1 / 13$ & \\
\hline Autre & $2 / 4$ & $5 / 13$ & \\
\hline Hémorragie du post-partum & $5(25.0)$ & $4(8.2)$ & 0.06 \\
\hline Nalador & 5 & 3 & \\
\hline Transfusion & 2 & 1 & \\
\hline Capitonnage & 0 & 0 & \\
\hline Ligature & 0 & 0 & \\
\hline Embolisation & 0 & 0 & \\
\hline Périnée & & & 0.29 \\
\hline Intact & $7(43.7)$ & $23(65.7)$ & \\
\hline Déchirure & $5(31.3)$ & $8(22.9)$ & \\
\hline Episiotomie & $3(18.8)$ & $4(11.4)$ & \\
\hline Périnée complet & $1(6.2)$ & $0(0.0)$ & \\
\hline Endométrite du post-partum & $0(0.0)$ & $0(0.0)$ & \\
\hline
\end{tabular}

${ }^{I} L A$ : liquide amniotique, ${ }^{2} A R C F$ : anomalie du rythme cardiaque foetal 
Tableau 5 : Caractéristiques des nouveau-nés à la naissance

\begin{tabular}{|c|c|c|c|}
\hline & $\begin{array}{c}\text { Cerclage avec ATCD } \\
\mathrm{N}=20\end{array}$ & $\begin{array}{l}\text { Pas de cerclage } \\
\quad N=49\end{array}$ & $\mathrm{p}$ \\
\hline & $\mathrm{N}(\%)$ & $\mathrm{N}(\%)$ & \\
\hline Sexe J1 & & & 0.92 \\
\hline Féminin & $11(55.0)$ & $26(53.1)$ & \\
\hline Masculin & $9(45.0)$ & $23(46.9)$ & \\
\hline Sexe $\mathbf{J} 2$ & & & 0.51 \\
\hline Féminin & $10(50.0)$ & $23(46.9)$ & \\
\hline Masculin & $10(50.0)$ & $26(53.1)$ & \\
\hline Poids J1 (moyenne \pm DS) & $1811.1 \pm 920.0$ & $1857.5 \pm 816.6$ & 0.83 \\
\hline$<10^{\text {ème }}$ percentile & $0(0.0)$ & $8(16.3)$ & 0.06 \\
\hline Poids J2 (moyenne \pm DS) & $1789.1 \pm 943.1$ & $1805.2 \pm 793.1$ & 0.94 \\
\hline$<10^{\text {ème }}$ percentile & $3(15.0)$ & $9(18.4)$ & 0.74 \\
\hline \multicolumn{4}{|l|}{ pH J1 } \\
\hline$<7.20$ & $0(0.0)$ & $2(5.1)$ & 0.36 \\
\hline$<7.10$ & $0(0.0)$ & $1(2.6)$ & 0.52 \\
\hline$<7.00$ & $0(0.0)$ & $1(2.6)$ & 0.52 \\
\hline \multicolumn{4}{|l|}{$\mathrm{pH} \mathrm{J} 2$} \\
\hline$<7.20$ & $2(11.8)$ & $4(10.5)$ & 0.89 \\
\hline$<7.10$ & $0(0.0)$ & $1(2.6)$ & 0.50 \\
\hline$<7.00$ & $0(0.0)$ & $0(0.0)$ & \\
\hline APGAR < 4 à 5 minutes $\mathrm{J} 1$ & $2(12.5)$ & $1(2.5)$ & 0.13 \\
\hline APGAR < 4 à 5 minutes $\mathrm{J} 2$ & $2(11.8)$ & $2(5.1)$ & 0.38 \\
\hline \multicolumn{4}{|l|}{ Réanimation néonatale J1 } \\
\hline Assistance ventilatoire & $6(30.0)$ & $11(22.5)$ & 0.51 \\
\hline Intubation & $2(10.0)$ & $2(4.1)$ & 0.34 \\
\hline \multicolumn{4}{|l|}{ Réanimation néonatale $\mathrm{J} 2$} \\
\hline Assistance ventilatoire & $9(45.0)$ & $15(30.6)$ & 0.26 \\
\hline Intubation & $2(10.0)$ & $2(4.1)$ & 0.34 \\
\hline
\end{tabular}

